\title{
Verification of smoking history in patients after infarction using urinary nicotine and cotinine measurements
}

\author{
R G WILCOX, J HUGHES, J ROLAND
}

British Medical fournal, 1979, 2, 1026-1028

\section{Summary and conclusions}

Urinary concentrations of nicotine and its major metabolite cotinine were measured in volunteers whose smoking habits were known to test the reliability of the measurements as indicators of current smoking. In the non-smokers detectable concentrations were always below the confidence limits set for the method, while in smokers the concentrations were always above these limits. After subjects stopped smoking cotinine appeared in the urine for longer than nicotine and was still detectable at least 36 hours after the last cigarette had been smoked. When this method was used to verify the smoking histories given by patients attending an infarction clinic it was estimated that $\mathbf{4 6 - 5 3 \%}$ of previous smokers had actually stopped smoking compared with the $63 \%$ who said that they had done so.

It is suggested that simultaneous assays of urinary nicotine and cotinine may be a useful means of verifying patients' current smoking habits.

\section{Introduction}

The health hazards attributed to smoking and the benefits that accrue from giving up have received considerable attention. ${ }^{1-5}$ Unfortunately, patients may not always be truthful in declaring their current smoking habits, ${ }^{6}$ so the real value of stopping smoking may be underestimated. Objective methods of validating patients' smoking histories have been sought. Determining plasma concentrations of thiocyanate, which has a long half life and is relatively easily measured, is currently a favoured method, although there is considerable overlap between nonsmokers and those smoking less than 10 cigarettes a day. ${ }^{7}$ Blood carboxyhaemoglobin concentrations have proved to be not sufficiently discriminating, and urinary nicotine measurements have been suggested as providing a more accurate assessment. ${ }^{8}$ Nicotine is rapidly absorbed through the lungs and reaches a peak concentration in the urine within 15 minutes after a cigarette has been smoked. The amount of unchanged nicotine excreted, however, is small, most of the excretion being in the form of its major metabolite cotinine. After smoking the peak urinary cotinine excretion occurs at about two hours and its clearance is slow. ${ }^{9}$

We have investigated the value of measuring both urine nicotine and cotinine concentrations as an index of current smoking and have used nicotine and cotinine measurements to verify the smoking histories given by patients attending a postinfarction clinic.

University Hospital, Nottingham NG7 2UH

R G WILCOX, MRCP, lecturer in medicine

J HUGHES, FIMLS, chief medical laboratory scientific officer, department of clinical chemistry

City Hospital, Nottingham

J ROLAND, MRCP, medical registrar

\section{Subjects and methods}

We collected random samples of urine for nicotine and cotinine measurements from 104 volunteers (46 non-smokers and 58 smokers) whose smoking history we considered to be reliable. To study the timepattern of nicotine and cotinine excretion we also collected samples of urine at about $0800,1200,1600$, and 2200 from 13 habitual smokers, who continued to smoke normally for the first 24 hours and then attempted to abstain during the next 36 hours. We then collected urine samples from 85 patients who attended our infarction clinic during the study period, and at that visit we recorded the patients' declared current smoking habits. They had all smoked regularly up to the time of their illness. We compared the previous and current smoking state of 305 consecutive patients attending the infarction clinic as part of secondary prevention drug trials. The smokers among them had been strongly advised to stop smoking during their initial admission. This advice was reiterated at each clinic visit to those who continued to smoke, whereas those who said they had stopped were commended and advised never to start smoking again.

The concentrations of nicotine and its metabolite cotinine in the urine samples were measured by $\mathrm{JH}$ using a modification of the method described by Kivela ${ }^{10}$ and without knowing the smoking history of the patients from whom the samples derived. The assays were done in batches within one week after collecting the specimen. To $20 \mathrm{ml}$ urine was added $4 \mathrm{~mol}$ sodium hydroxide $/ 1$ and $0.5 \mathrm{ml}$ internal standard solution containing $0.02 \mathrm{mg}$ methyprylone in chloroform. Chloroform, $7 \mathrm{ml}$, was then added and mixed for 15 minutes. The layers were separated by centrifuging lightly for one minute. The chloroform extract was transferred to a tapered centrifuge tube and evaporated down to approximately $50 \mu \mathrm{l}$ at room temperature under vacuum, and $3 \mu \mathrm{l}$ of the concentrated extract was injected into a Pye 104 gas-liquid chromatograph fitted with a flame ionisation detector. The column was $10 \%$ human chorionic gonadotrophin (Apiezon $\mathrm{L}$ ) on Diatonite CQ 80-100 mesh (Pye Unicam), column temperature $200^{\circ} \mathrm{C}$, carrier gas (nitrogen) flow rate $70 \mathrm{ml} / \mathrm{min}$. Quantitative results were obtained by comparing the peak height ratios of nicotine:internal standard or cotinine : internal standard against those of aqueous standards treated as urine. The sensitivity of the method was established using standard solutions of decreasing concentration. The confidence detection concentrations for nicotine and cotinine were found to be $0.12 \mu \mathrm{mol} / 1(2 \mu \mathrm{g} / 100 \mathrm{ml})$ and $0.57 \mu \mathrm{mol} / 1(10 \mu \mathrm{g} / 100 \mathrm{ml})$ respectively.

The creatinine concentration of each urine sample was also measured in order to compare cotinine:creatinine ratios between individuals smoking the same number of cigarettes each day.

\section{Results}

\section{VALIDATION OF METHOD}

We collected 68 random urine samples from the 46 non-smoking volunteers (never smoked, 42 ; not smoked for at least one year, four). None of the samples contained any nicotine, and although four $(6 \%)$ contained cotinine, the concentrations were below the confidence limit of our method. Thus no non-smoker had either a urinary nicotine or a cotinine concentration above the confidence limits set by our evaluation of the method.

We collected 188 urine samples from the 58 volunteers who smoked regularly. Of these, 29 smoked a constant number of cigarettes each day, ranging from three to 45 (mean 27 cigarettes/day). The other 29 volunteers were trying to reduce their intake and smoked a varying number of cigarettes each day. All the urine samples contained cotinine in concentrations detectable with confidence by our method, the mean $( \pm S E$ of mean) value being $6.04 \pm 0.73 \mu \mathrm{mol} / 1(106 \pm 13$ $\mu \mathrm{g} / 100 \mathrm{ml})$. Similarly they all contained nicotine in excess of the confidence level for our method, the mean value being $7 \cdot 96 \pm 1 \cdot 11$ $\mu \mathrm{mol} / 1(133 \pm 19 \mu \mathrm{g} / 100 \mathrm{ml})$. In this smoking group as a whole there was no correlation between the declared number of cigarettes smoked and the urinary nicotine and cotinine concentrations. In the subgroup 
with a constant daily cigarette consumption, however, the number of cigarettes smoked correlated strongly with the urinary cotinine: creatinine ratio (fig 1 ).

Thirteen of the smoking volunteers with constant smoking habits then attempted to abstain from smoking for the next 36 hours, but only nine succeeded. Figure 2 shows their mean urinary nicotine and cotinine concentrations for the 24 -hour period of normal smoking followed by the next 36 hours of abstinence. This indicates the relatively slower rate of disappearance of cotinine from the urine in comparison with nicotine. All these volunteers regularly smoked six or more cigarettes daily, and eight of them had confidently detectable urinary cotinine concentrations at least 36 hours after smoking their last cigarette, the one negative result occurring in the subject who smoked the least. Nicotine was found in the urine in only two subjects at 2200 on day 2 , and in one subject at 0800 on day 3.

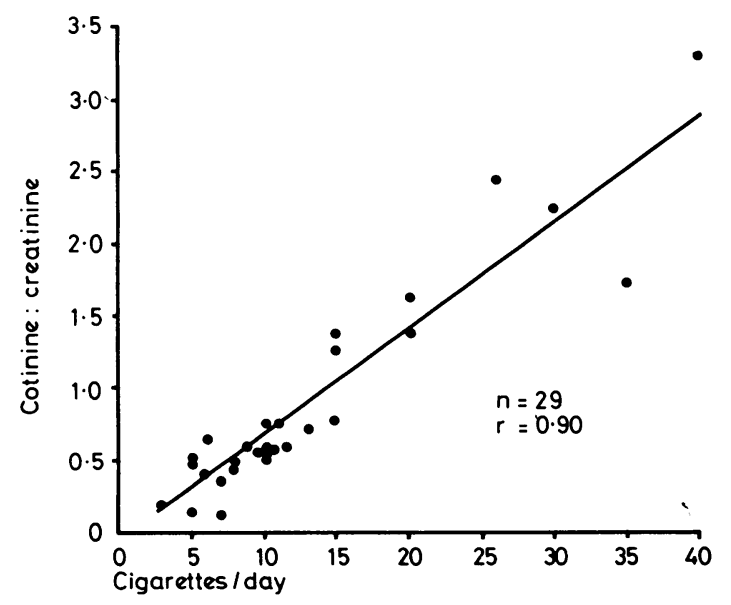

FIG 1-Correlation between urinary cotinine:creatinine ( $\mu \mathrm{mol}: \mathrm{mmol}$ ) ratios and number of cigarettes smoked in 29 regular smokers.

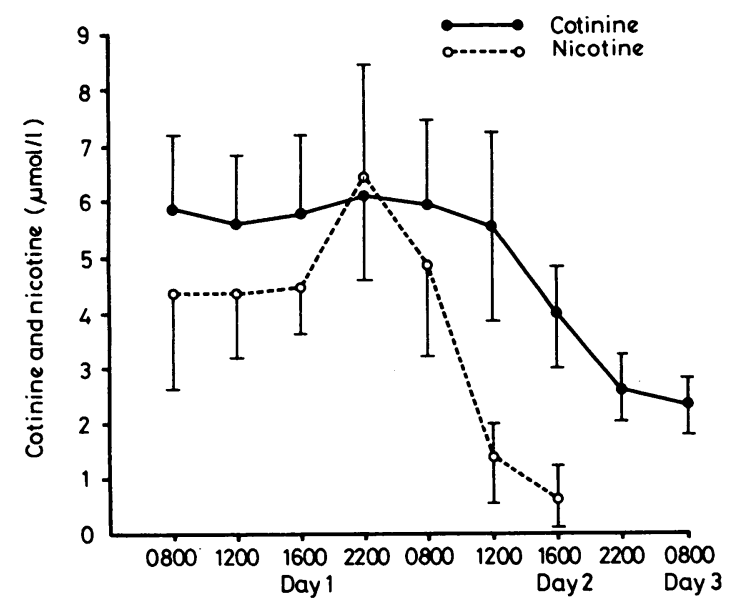

FIG 2-Disappearance of nicotine and cotinine from urine in nine patients who stopped smoking at about 2200 on day 1 . Values are means $\pm \mathrm{SE}$ of mean; $\mathrm{n}=9$.

USING METHOD TO CHECK SMOKING HISTORY IN PATIENTS AFTER INFARCTION

Of the 85 subjects attending the infarction clinic who had been regular smokers up to the time of their illness, $36(42 \%)$ said that they were still smoking and all of these had easily detectable urinary nicotine and cotinine concentrations. The remaining $49(58 \%)$ patients said that they had stopped smoking, and in 36 of these we could not detect either nicotine or cotinine in their urine. In eight patients, however, both nicotine and cotinine could be confidently detected in the urine, while in the remaining five patients cotinine and nicotine were detectable but in concentrations below the confidence limits of our method. This suggests that eight to 13 of these 49 declared ex-smokers were still smoking, a deception rate of about $16-26 \%$.

Of the 305 consecutive patients (254 men, 51 women) attending the infarction clinic during this survey, $208(68 \%)$ had been regular smokers up to the time of their illness, $85(28 \%)$ had never smoked, and $12(4 \%)$ had not smoked for at least one year before their illness.

TABLE I-Changes in smoking habits of 208 smokers after heart attack according to previous consumption

\begin{tabular}{lllllll}
\hline & \multicolumn{5}{c}{ No of cigarettes previously smoked/day } \\
\cline { 2 - 6 } & $\leqslant 10$ & -20 & -30 & -40 & $>40$ \\
\hline No of patients & 43 & 70 & 39 & 31 & 25 \\
No (\%) continuing smoking & $16(37)$ & $26(37)$ & $12(31)$ & $15(48)$ & $8(32)$ \\
Mean No of cigarettes currently & $\begin{array}{l}\text { smoked/day } \\
\text { No }(\%) \text { stopped smoking }\end{array}$ & 5 & 9 & 11 & 12 & 19 \\
& $27(63)$ & $44(63)$ & $27(69)$ & $16(52)$ & $17(68)$ \\
\hline
\end{tabular}

TABLE II-Changes in smoking habits according to time since heart attack

\begin{tabular}{lcccc}
\hline & \multicolumn{2}{c}{ No of months after heart attack } & \multirow{2}{*}{ Total } \\
\cline { 2 - 3 } & -3 & -9 & -18 & \\
\hline No of patients & 61 & 82 & 162 & 305 \\
No (\%) who smoked previously & $48(79)$ & $61(74)$ & $99(61)$ & 208 \\
No $(0 \%)$ who stopped & $29(60)$ & $37(61)$ & $65(66)$ & 131 \\
No $(\%)$ who reduced consumption & $15(31)$ & $20(33)$ & $25(25)$ & 60 \\
No $(\%)$ in whom no change & $4(8)$ & $4(7)$ & $9(9)$ & 17 \\
\hline
\end{tabular}

When asked about their current smoking habits $131(63 \%)$ of the previously regular smokers said that they had now stopped, and this reply was independent of their previous consumption of cigarettes (table I) or the timing of the survey in relation to the heart attack (table II). If the deception rate among this larger group of smokers is similar to that in the group whose urine was tested, however, the true percentage of previous smokers who actually had stopped smoking is likely to be between 46 and $53 \%$.

\section{Discussion}

The answer to the question "Are you still smoking cigarettes and if so how many ?" is likely to be influenced by the manner and circumstances in which it is asked. Burt et al $^{11}$ gave a group of patients who had had an infarction "intensive advice" with a "forceful approach" and found that $63 \%$ of previous smokers said they had stopped as compared with $27 \%$ who were given conventional advice. We gave our patients conventional but uncompromising advice to stop smoking and also found that $63 \%$ of previous smokers said they had done so, irrespective of previous cigarette consumption or the time since infarction. How is the accuracy of such statements best established?

When measuring the carboxyhaemoglobin ( $\mathrm{COHb}$ ) concentrations in venous blood Sillett et $a l^{6}$ found that $22-40 \%$ of alleged ex-smokers had concentrations above $1.7 \%$, their critical value based on measurements obtained in 161 known nonsmokers. No general agreement exists, however, on this critical value, and in that study five out of 86 patients who said they were still smoking had $\mathrm{COHb}$ concentrations below $1.7 \%$. Furthermore, $\mathrm{COHb}$ concentrations can be influenced by environmental pollution ${ }^{12}$ and passive smoking. ${ }^{13}$

Urinary nicotine measurements, too, are influenced by passive smoking under extreme experimental exposure, but, by using a method similar to that described here and incorporating a nitrogen detector, which increases the sensitivity considerably, Russell and Feyerabend ${ }^{8}$ found no overlap between smokers and non-smokers under normal conditions. In our study no nonsmoker had any detectable nicotine, although four had detectable cotinine but at concentrations below the confidence limit of our assay. In all the current smokers both nicotine and cotinine could be readily detected in their urine. 
In future studies we shall note the brand of cigarette smoked, for this will determine the nicotine yield per cigarette. This clearly will influence the correlation between urinary cotinine: creatinine ratios and the number of cigarettes smoked each day and, in the current study, may explain some of the scatter.

We suggest that simultaneous assays of urinary nicotine and cotinine could be a useful index of current smoking habits and that the slower disappearance of cotinine from the urine will facilitate identifying smokers who attempt to conceal their habit by abstaining from smoking on the day of their clinic visit.

We should like to thank Mr E J Collin, of John Player and Sons, Nottingham, for arranging the supplies of cotinine; Mr A Bridge, principal biochemist, University Hospital, Nottingham, for technical guidance; Professor J R A Mitchell for his helpful criticism; and Mrs Jayne Webster for typing the manuscript.

\section{References}

${ }^{1}$ Doll, R, and Hill, A B, National Cancer Institute Monographs, 1966, 19, 205.

${ }^{2}$ Hammond, E C, and Garfinkel, L, Archives of Environmental Health, 1969, 19, 167.

${ }^{3}$ Gordon, T, Kannel, W B, and McGee, D, Lancet, 1974, 2, 1345.

4 Wilhelmsson, C, et al, Lancet, 1975, 1, 415.

5 Doll, R, and Peto, R, British Medical fournal, 1976, 2, 1525.

${ }^{6}$ Sillett, R W, et al, British Medical fournal, 1978, 2, 1185.

7 Vogt, T M, et al, American fournal of Public Health, 1977, 67, 545.

${ }^{8}$ Russell, M A H, and Feyerabend, C, Lancet, 1975, 1, 179.

${ }^{9}$ Beckett, A H, and Triggs, E J, Nature, 1966, 211, 1415.

${ }^{10}$ Kivela, E W, in Manual of Toxicology, ed I Sunshine, p 252. Cleveland, Ohio, CRC Press Inc, 1971.

11 Burt, A, et al, Lancet, 1974, 1, 304.

12 Jones, R D, Commins, B T, and Cernik, A A, Lancet, 1972, 2, 302.

13 Russell, M A H, Cole, P V, and Brown, E, Lancet, 1973, 1, 576.

(Accepted 14 August 1979)

\title{
Return to work and quality of life after surgery for coronary artery disease
}

\author{
STEPHEN WESTABY， RALPH N SAPSFORD， HUGH H BENTALL
}

British Medical fournal, 1979, 2, 1028-1031

\section{Summary and conclusions}

Changes in work capability and quality of life were assessed retrospectively in 130 patients with ischaemic heart disease who had undergone aortocoronary bypass operations during 1976-7 because of medically uncontrollable angina.

A total of 85 patients $(65.4 \%)$ reported complete relief from angina six months after operation, though 12 later suffered a recurrence. Substantially fewer patients needed drugs after the operation. Before operation 9 out of 117 men fully employed at the onset of angina were working without restriction or doing lighter, fulltime work, 38 were at work but seriously incapacitated by angina, and 70 were forced to stop work. After operation 70 were working without restriction or engaged in lighter work, 15 were at work but still restricted by angina, and only 32 were forced to stop work. This result was highly significant $(P<0.001)$. These differences were even more pronounced in heavy manual workers, of whom none could work normally before operation, whereas 16 were working without restriction afterwards. Of patients wishing to engage in hobbies or sports, social activity, and sexual intercourse but were restricted before operation, about two-thirds could resume these activities afterwards.

Royal Postgraduate Medical School, Hammersmith Hospital, London W12 0HS

STEPHEN WESTABY, BSC, FRCS, registrar in cardiothoracic surgery (now locum senior registrar in cardiothoracic surgery, Harefield Hospital, Harefield, Middlesex)

RALPH N SAPSFORD, CHM, FRCS, consultant and senior lecturer in cardiothoracic surgery

HUGH H BENTALL, MB, FRCS, professor of cardiac surgery and honorary consultant thoracic surgeon
Coronary artery surgery provided dramatic symptomatic relief in up to $90 \%$ of patients and permitted rehabilitation and return to gainful employment irrespective of type of labour. The degree of symptomatic improvement and increase in exercise tolerance after successful surgery is usually far greater than occurs with any other form of treatment and directly improves quality of life and work capability.

\section{Introduction}

Coronary artery disease is a major cause of death and disability in men aged 35-65 years, who are traditionally regarded as the most economically productive members of society. ${ }^{1}$ Its manifestations include sudden death, myocardial infarction, heart failure, and angina pectoris. Although many patients respond to medical management, the development of aortocoronary bypass grafting using the saphenous vein over the past 10 years offers a new and increasingly acceptable approach, especially for the severely handicapped. Many large series have shown benefit in terms of pain relief, ${ }^{2}$ prevention of myocardial infarction, ${ }^{3}$ and improvement in ventricular function. ${ }^{4}$ There is also evidence of increased longevity. ${ }^{5}$ Although early reports showed fewer patients working after operation than before, ${ }^{6}$ ? later results were encouraging. ${ }^{8} 9$

We have investigated the quality of life and work capability of patients before and after operations performed in this hospital during 1976-7.

\section{Patients and methods}

Patients were selected for operation after failure to control their angina by standard medical treatment and elimination of risk factors. A total of 156 patients underwent coronary artery bypass grafting during the two years, of whom nine had been operated on before. Three of the patients also underwent resection of a left ventricular aneurysm. Of the 14 known deaths, two occurred in hospital and 12 after 30 days. Nine patients died of their disease without returning to work, one died after pneumonectomy for carcinoma of the bronchus, and another died after an aortofemoral bypass operation for peripheral 